\title{
Crystallographic studies of sero-specific Dengue ED3 immune response
}

\author{
Manjiri R. Kulkarni ${ }^{1}$, Monirul Islam ${ }^{2}$, Nobutaka Numoto ${ }^{3}$, Montasir Elahi ${ }^{4}$, Nobutoshi Ito ${ }^{3}$ and
}

\section{Yutaka Kuroda $^{4}$}

${ }^{1}$ Department Of Comparative Biosciences, University of Illinois, Urbana-Champaign, Urbana, United States, ${ }^{2}$ Department of Biochemistry and Molecular Biology, University of Chittagong, Chittagong,

Bangladesh, ${ }^{3}$ Department of Structural Biology, Medical Research Institute, Tokyo Medical and Dental University, Tokyo, Japan, ${ }^{4}$ Department of Biotechnology and Life Sciences, Tokyo University of Agriculture and Technology, Tokyo, Japan

\section{E-mail: mrk@illinois.edu}

Dengue (DEN) fever is a re-emerging tropical disease. Although, the four serotypes (DEN1-DEN4) have $70-80 \%$ sequence and high structural similarity, slight variations present in different serotypes are responsible for the sero-specific nature of individual serotype, which cause a life-threatening dengue hemorrhagic fever and dengue shock syndrome. Here, we aim at characterizing the sero-specificity of the immune response against DEN3 and DEN4, envelope domain 3 (ED3) through structural and biophysical analysis. ED3 contains the two major putative epitopes (E1 and E2) [1] and is a highly suitable model protein for examining the molecular determinants of a virus' sero-specificity. We designed six epitope grafted ED3 variants where the surface-exposed epitope residues of E1 and E2 from DEN3 ED3 were switched to those of DEN4 ED3 and vice versa individually and in combination by hypothesizing that sero-specificity would be transferred by switching the epitope residues. Synthetic genes encoding the ED3 sequences of DEN3 and DEN4 were cloned into a pET15b vector, overexpressed and proteins were purified. Immunizing Swiss-Albino mice produced anti-DEN3 ED3 and anti-DEN4 ED3 sera. Interaction strengths were monitored by ELISA using purified ED3s and both anti-sera. Finally, crystal structure of DEN4 ED3's epitope grafted mutant where E2 residues are mutated from DEN3 ED3 (DEN4_E2 ${ }^{\text {DEN3 }}$ hereafter, PDB ID: 4X42) was determined [2] by molecular replacement using DEN4 ED3 structure. Interaction patterns were further confirmed using highresolution crystal structures of DEN3 ED3 (PDB ID: 3VTT), DEN4 ED3 (PDB ID: 4WE1) [3] and DEN4_E2 ${ }^{\text {DEN3 }}$ plus electrostatic surface potentials (ESPs) of other modeled grafted mutants. Mutants that were difficult to crystallize were modeled using DEN3 ED3 and DEN4 ED3 as a template in respective serotype mutant. We found individual protein domain ED3 gives highly sero-specific immune responses in mice. Reactivity against epitope-grafted mutants indicated that E1 in DEN4 ED3 and E2 in DEN3 ED3 play a comparatively major role in determining sero- specificity. Most importantly, crystal structures and ESPs gave an additional insight into sero-specific nature of E1 and E2. The structure of DEN4_E2 ${ }^{\text {DEN3 }}$ was essentially identical to that of DEN4 ED3 (RMSD: $0.33 \AA$ ) despite E2's residues being substituted from DEN3 ED3, stating that E1 plays a major role in serospecificity of DEN4 ED3. Moreover, similar structures and ESPs of DEN4 ED3 and DEN4_E2 ${ }^{\text {DEN3 }}$ confirmed the similarity of interaction patterns shown by these two antigens against both anti-sera in ELISA. To sum up, detailed examination of the structures and electrostatic surfaces nevertheless provided insights into the sero-specificity of DEN ED3s that were unnoticed from sequence. This study was partly supported by a Joint Usage/Research Program at the Medical Research Institute of Tokyo Medical and Dental University and a Japanese government (Monbukagakusho: MEXT) PhD scholarship to M.R.K. X-ray diffraction data were collected at the Photon Factory, KEK (Tsukuba, Japan) under project numbers 2013G133 and 2015G128.

[1] Lisova. O. et al. (2007) J. Gen. Virol. 88, 2387-2397.

[2] Kulkarni. M. et al. (2015) Biochimica Biophysica Acta 1854, 1438-1443.

[3] Elahi. M. et al. (2013) Proteins 81, 1090-1095. 